\title{
DESIGN OF ADVANCED TARGETING COST FUNCTION FOR EVOLUTIONARY OPTIMIZATION OF CHAOS CONTROL
}

\author{
Roman Senkerik \\ Ivan Zelinka \\ Zuzana Oplatkova \\ Department of Applied Informatics \\ Faculty of Applied Informatics, Tomas Bata University in Zlin \\ Nad Stranemi 4511, Zlin 762 72, Czech Republic \\ E-mail: \{senkerik, zelinka, oplatkova\}@fai.utb.cz
}

\section{KEYWORDS}

Chaos, control, optimization, evolutionary algorithms

\section{ABSTRACT}

This research deals with the optimization of the control of chaos by means of evolutionary algorithms. The main aim of this work is to show that powerful optimizing tools like evolutionary algorithms can in reality be used for the optimization of deterministic chaos control. This work is aimed on an explanation of how to use evolutionary algorithms (EAs) and how to properly define the advanced targeting cost function (CF) securing very fast and precise stabilization of desired state for any initial conditions. As a model of deterministic chaotic system, the two dimensional Henon map was used. The evolutionary algorithm SelfOrganizing Migrating Algorithm (SOMA) was used in four versions. For each version, repeated simulations were conducted to outline the effectiveness and robustness of used method and targeting $\mathrm{CF}$.
\end{abstract}

\section{INTRODUCTION}

The question of targeting (faster stabilization) with application to chaos control has attracted researchers since the first method for controlling of chaos was developed. The several first approaches for targeting have used special versions of OGY control scheme (Kostelich et al., 1993; Bolt and Kostelich, 1998) or collecting of information about trajectories, which fall close to desired state (Bird and Aston, 1998). Later, alot of methods were based on adaptive approach (Ramaswamy et al., 1998), center manifold targeting (Starrett, 2002) or neural networks (Iplikci and Denizhan, 2001; Iplikci and Denizhan, 2003).

Currently, evolutionary algorithms (EA) are known as powerful tools for almost any difficult and complex optimization problem. But the quality of obtained results through optimization mostly depends on proper design of the used cost function, especially when the EAs are used for optimization of chaos control. The results of numerous simulations lends weight to the argument that deterministic chaos in general and also any technique to control of chaos are sensitive to parameter setting, initial conditions and in the case of optimization, they are also extremely sensitive to the construction of used cost function.

This research utilized the Pyragas's delayed feedback control technique (Just 1999, Pyragas 1992). Unlike the original OGY control method (Ott, et al. 1990) it can be simply considered as a targeting and stabilizing algorithm together in one package (Kwon 1999). Another big advantage of Pyragas method is the amount of accessible control parameters. This is very advantageous for successful use of optimization of parameters setting by means of EA, leading to improvement of system behavior and better and faster stabilization to the desired periodic orbits. Some research in this field has been recently done using the evolutionary algorithms for optimization of local control of chaos (Richter and Reinschke, 2000; Richter, 2002). The control law in this work is based on the Pyragas method: Extended delay feedback control ETDAS (Pyragas 1995).

This research is concerned with the investigation of the design of the advanced targeting cost functions securing the stabilization to desired UPO (unstable periodic orbit) for any initial conditions. This work presents a accumulation of research (Zelinka et al., 2007) and also collates and elaborates the experiences with application of EA to chaos control (Senkerik et al. 2006; Senkerik et al. 2007) in order to reach the better results and decrease the influence of negative phenomenon which can occur in such a challenging task, which chaos control is.

\section{PROBLEM DESIGN}

\section{Problem selection and case studies}

The chosen example of a chaotic system was the two dimensional Henon map in form (1).

$$
\begin{aligned}
& x_{n+1}=a-x_{n}^{2}+b y_{n} \\
& y_{n+1}=x_{n}
\end{aligned}
$$

This work primarily consists of three case studies. All of them are focused on an estimation of three accessible control parameters for EDTAS control method to 
stabilize desired UPO, and a comparison of obtained results for used cost function. Desired UPOs are the following: $\mathrm{p}-1$ (a fixed point) in the first case, $\mathrm{p}-2$ in the second case and p-4 in the last case. All simulations were 50 times repeated for each EA version. The control method - ETDAS in the discrete form suitable for two dimensional Henon map has the form (2).

$$
\begin{gathered}
x_{n+1}=a-x_{n}^{2}+b y_{n}+F_{n} \\
F_{n}=K\left[(1-R) S_{n-m}-x_{n}\right] \\
S_{n}=x_{n}+R S_{n-m}
\end{gathered}
$$

where $K$ and $R$ are adjustable constants, $F$ is the perturbation, $S$ is given by a delay equation utilizing previous states of the system and $m$ is the period of $m$ periodic orbit to be stabilized. The perturbation $F_{n}$ in equations (2) may have an arbitrarily large value, which can cause diverging of the system outside the interval $\{-1.5,1.5\}$. Therefore, $F_{n}$ should have a value between $-F_{\max }, F_{\max }$ and EA should find an appropriate value of this limitation to avoid diverging of the system.

\section{The basic cost function}

The proposal of the basic cost function (CF) is in general based on the simplest $\mathrm{CF}$, which could be used only for the stabilization of $\mathrm{p}-1$ orbit. The idea was to minimize the area created by the difference between the required state and the real system output on the whole simulation interval $-\tau_{\mathrm{i}}$.

But another cost function had to be used for stabilizing of the higher periodic orbit. It was synthesized from the simple CF and other terms were added. In this case, it is not possible to use the simple rule of minimizing the area created by the difference between the required and actual state on the whole simulation interval $-\tau_{\mathrm{i}}$, due to the many serious reasons, for example: degrading of the possible best solution by phase shift of periodic orbit.

This CF, is in general based on searching for desired stabilized periodic orbit and thereafter calculation of the difference between desired and found actual periodic orbit on the short time interval $-\tau_{\mathrm{s}}$ (approx. $20-50$ iterations) from the point, where the first min. value of difference between desired and actual system output is found. Such a design of CF should secure the successful stabilization of higher periodic orbit anywise phase shifted.

Furthermore, because of CF values being very close to zero, this CF also allows using of decision rule avoiding very time demanding simulations. This rule stops EA immediately, when the first individual with good parameter structure is reached, thus the value of $\mathrm{CF}$ is lower then the acceptable $\left(\mathrm{CF}_{\mathrm{acc}}\right)$ one. Typically $\mathrm{CF}_{\mathrm{acc}}=$ 0.001 at time interval $\tau_{\mathrm{s}}=20$ iterations, thus the difference between desired and actual output has value 0.0005 per iteration - i.e. successful stabilization for used control technique. This $\mathrm{CF}$ was also used for $\mathrm{p}-1$ orbit. The $\mathrm{CF}_{\text {Basic }}$ has the form (3).
$C F_{\text {Basic }}=$ penalization $_{1}+\sum_{t=\tau 1}^{\tau 2}\left|T S_{t}-A S_{t}\right|$

where: TS - target state, AS - actual state

$\tau_{1}$ - the first min. value of difference between TS and AS

$\tau_{2}-$ the end of optimalizing interval $\left(\tau_{1}+\tau_{\mathrm{s}}\right)$

penalization $_{l}=0$ if $\tau_{\mathrm{i}}-\tau_{2} \geq \tau_{\mathrm{s}}$;

penalization $_{I}=10^{*}\left(\tau_{\mathrm{i}}-\tau_{2}\right)$ if $\tau_{\mathrm{i}}-\tau_{2}<\tau_{\mathrm{s}}$

(i.e. late stabilization)

\section{The advanced targeting cost function}

It was necessary to modify the definition of CF in order to decrease the average number of iteration required for the successful stabilization and avoidance of any associated problem. The $\mathrm{CF}_{\mathrm{Basic}}$ is suitable for adding some term of penalization for slowly stabilizing solutions, thus it was modified for the use of all required UPOs. The $\mathrm{CF}$ value is multiplied by the number of iterations $(N I)$ of the first found minimal value of difference between desired and actual system output (i.e. the beginning of fully stabilized UPO). To avoid problems associated with $\mathrm{CF}$ returning value 0 and to put the penalization to similar level as the nonpenalized $\mathrm{CF}$ value, the small constant $(S C)$ is added to $\mathrm{CF}$ value before penalization (multiplying by $N I$ ).

Generally, there exist two possible approaches for defining the $\mathrm{SC}$ value. The first one capitalizes the previous simulation results with $\mathrm{CF}$ basic and experiences, whereas the second approach uses the automatically computed value.

The next two proposals of CF design are based on the second approach, which should avoid any problems associated with defining the value of small constant, SC in advance (especially for stabilization of higher periodic orbit). The SC value (5) is computed with the aid of power of non-penalized basic part of CF (4).

$\operatorname{Exp} C F=\log _{10}\left(\sum_{t=\tau 1}^{\tau 2}\left|T S_{t}-A S_{t}\right|+10^{-15}\right)$

$S C=10^{\mathrm{ExpCF}}$

In general, there exists two possible ways for applying the multiplication by number of iterations required for stabilization $(N I)$. The first version of final design of targeting $\mathrm{CF}\left(\mathrm{CF}_{\mathrm{T} 1}\right.$ - ADV $)$ has the form (6). Here the sum of basic part of $\mathrm{CF}$ and automatically computed $S C$ is multiplied by NI. Finally, to avoid the problems with fast stabilization, only for limited range of initial conditions, the final CF value is computed as a sum of $n$ repeated simulations for different initial conditions. Consequently, the EA should find the robust solutions securing the fast targeting into desired behavior of system for almost any initial conditions. 


$$
C F_{T 1-A d v}=\sum_{1}^{n} N I\left(S C+\text { penalization } 1+\sum_{t=\tau 1}^{\tau 2}\left|T S_{t}-A S_{t}\right|\right)
$$

where: $x_{\text {initial }}$ is from the range $0.05-0.95$ and uses step 0.1 .

In the second version of targeting $\mathrm{CF}\left(\mathrm{CF}_{\mathrm{T} 2}\right.$ - $\left.\mathrm{ADV}\right)$, there is only a slight change in comparison with the previous proposal. Here the number of steps for stabilization $(N I)$ multiplies only the small constant $(S C)$ which is counted in the same way as in the previous case (5). This version of targeting $\mathrm{CF}\left(\mathrm{CF}_{\mathrm{T} 2}\right.$ - ADV $)$ has the form (7).

$$
C F_{T 2-A d v}=\sum_{1}^{n}\left((N I \cdot S C)+\text { penalization } 1+\sum_{t=\tau 1}^{\tau 2}\left|T S_{t}-A S_{t}\right|\right)
$$

\section{Graphical CF Overview}

The difference between the proposed CFs can be clearly seen in Figures 1 and 2, which shows the dependence of $\mathrm{CF}$ values on the adjustable parameter $K$. Remaining parameters were set at the best values reached in optimizations; consequently the two-dimensional diagram always shows the section of global minimum. From these figures, it is obvious as to how a small change in the CF design can influence the nonlinearity and unpredictability of CF surface.

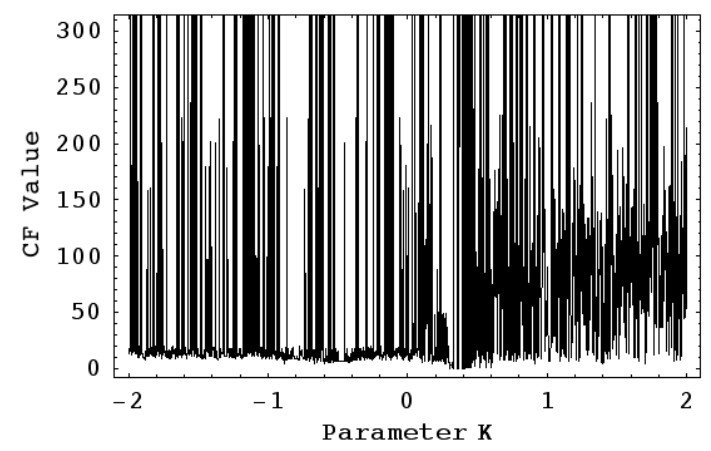

Fig. 1. Dependence of CF value on parameters $K$ for p-2 orbit, $x_{\text {initial }}=0.7, \mathrm{CF}_{\mathrm{T} 1-\mathrm{ADV}}, R=0.13, F_{\max }=0.12$

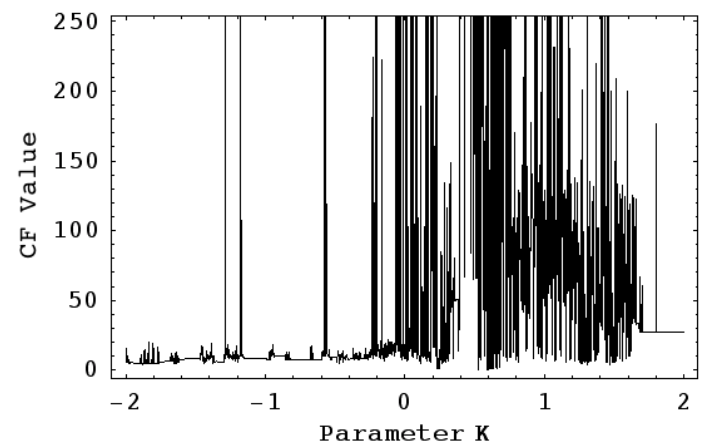

Fig. 2. Dependence of CF value on parameters $K$ for p-2 orbit, $x_{\text {initial }}=0.7, \mathrm{CF}_{\mathrm{T} 2-\mathrm{ADV}}, R=0.35, F_{\max }=0.18$

\section{Optimization algorithms}

For the experiments described here, stochastic optimization algorithm SOMA (Zelinka, 2004), has been used. It was chosen because it has been proven that this algorithm has the ability to converge towards the global optimum. SOMA works with groups of individuals (population) whose behavior can be described as a competitive - cooperative strategy. The construction of a new population of individuals is not based on evolutionary principles (two parents produce offspring) but on the behavior of social group, e.g. a herd of animals looking for food. This algorithm can be classified as an algorithm of a social environment. To the same group of algorithms, particle swarm algorithm can also be put in, sometimes called swarm intelligence. In the case of SOMA, no velocity vector works as in particle swarm algorithm, only the position of individuals in the search space is changed during one generation, here called 'Migration loop'.

The rules are as follows: In every migration loop, the best individual is chosen, i.e. individual with the minimum cost value, which is called "Leader". An active individual from the population moves in the direction towards Leader in the search space. At the end of the movement, the position of the individual with minimum cost value is chosen. If the cost value of the new position is better than the cost value of an individual from the old population, the new individual appears in new population. Otherwise the old one remains for the next migration loop.

\section{EXPERIMENTAL RESULTS}

Four versions of SOMA were used for all simulations. (See Table 1). See also Table 2 for parameter set up of EA. Parameters for the optimizing algorithm were set up in such a way in order to reach the same value of maximal $\mathrm{CF}$ evaluations for all used versions. Each version of SOMA has been applied 50 times in order to find the actual optimum.

The primary aim here is not to show which version is better or worse but to show that the EA can in reality be used for deterministic chaos control when the targeting cost function is properly defined.

All results are shown only for variable $x$ of two dimensional Henon map because of its form (1), where the variable $y$ has the same values as variable $x$ but it is only phase shifted.

Here is the list of desired UPOs:.

Henon Map with $a=1.2$ and $b=0.3$ :

p-1 (fixed point): $x_{F}=0.8$

p-2 orbit: $x_{1}=-0.562414, x_{2}=1.26241$

p-4 orbit: $x_{1}=0.139, x_{2}=1.4495, x_{3}=-0.8595, x_{4}=0.8962$

The optimization interval for $\mathrm{p}-1$ orbit was $\tau_{\mathrm{i}}=100$ iterations, for higher periodic orbits (p-2 and $\mathrm{p}-4)$ it was mostly $\tau_{\mathrm{i}}=150$ iterations. 
The ranges of all estimated parameters were these: $-2 \leq K \leq 2,0 \leq F_{\max } \leq 0.5$ and $0 \leq R \leq 0.5$

Table 1: Used versions of SOMA

\begin{tabular}{|c|c|}
\hline Index & Algorithm / Version \\
\hline 1 & SOMA AllToOne \\
\hline 2 & SOMA AllToRandom \\
\hline 3 & SOMA AllToAll \\
\hline 4 & SOMA AllToAllAdaptive \\
\hline
\end{tabular}

Table 2: Parameter Settings for SOMA

\begin{tabular}{|l|c|c|}
\hline \multicolumn{1}{|c|}{ Parameter } & ATO / ATR & ATA / ATAA \\
\hline PathLength & 3 & 3 \\
\hline Step & 0.33 & 0.33 \\
\hline PRT & 0.1 & 0.1 \\
\hline PopSize & 25 & 10 \\
\hline Migrations & 25 & 7 \\
\hline $\begin{array}{l}\text { Max. CF } \\
\text { Evaluations (CFE) }\end{array}$ & 5400 & 5670 \\
\hline
\end{tabular}

The best solution for each version of SOMA are shown in Tables $3-5$ together with other optimization results like average number of iterations required for successful stabilization for 50 repeated simulations (Avg. IStab). The figures $3-11$ shows the simulation of the best individual solutions for the uniformly distributed initial conditions in the range $0<x_{\text {initial }}<1$, and 100 samples were used in this kind of simulation

\section{Control of chaos, p-1 orbit}

The first case is focused on the stabilization of $\mathrm{p}-1$ orbit. For the best individual solutions given by $\mathrm{CF}_{\mathrm{T1}-\mathrm{ADV}}$ (SOMA ATAA) and $\mathrm{CF}_{\mathrm{T} 2 \text {-ADV }}$ (SOMA ATAA), please refer to Table 3. From the series of complex simulations depicted in Figures $3-5$, it is obvious, that the occurrence of the phenomenon, when some best individual solutions are not suitable for complex simulation with wide range of initial conditions, whereas the remaining best solutions give excellent results, was successfully suppressed by means of usage of advanced targeting cost functions. The example of this negative phenomenon is represented by simulation of results given by $\mathrm{CF}_{\text {Basic }}$. Based on obtained results, it may be stated that the control parameters estimated in the optimizations ensured very fast and precise reaching of a desired state for any initial conditions.

Table 3: Results for $\mathrm{p}-1$ orbit, $\mathrm{CF}_{\mathrm{T} 1-\mathrm{ADV}}$ and $\mathrm{CF}_{\mathrm{T} 2-\mathrm{ADV}}$

\begin{tabular}{|c|c|c|}
\hline CF Version & CF $_{\mathrm{T} 1-\mathrm{ADV}}$ & $\mathrm{CF}_{\mathrm{T} 2-\mathrm{ADV}}$ \\
\hline$K$ & -0.9977 & -0.8575 \\
\hline$F_{\max }$ & 0.4382 & 0.4688 \\
\hline$R$ & 0.3837 & 0.2148 \\
\hline CF Val. & $\mathbf{9 . 1 3 \mathbf { 1 0 } ^ { - 1 4 }}$ & $\mathbf{3 . 8 6} \mathbf{1 0}^{-\mathbf{1 4}}$ \\
\hline Avg. IStab & 56 & 41 \\
\hline
\end{tabular}

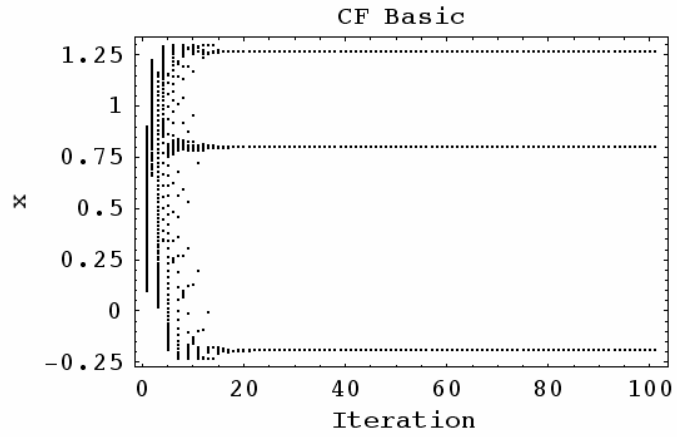

Fig. 3. Best solution: $\mathrm{p}-1$ orbit, $\mathrm{CF}_{\text {Basic }}$, SOMA ATA, complex simulation with $0<x_{\text {initial }}<1,100$ samples

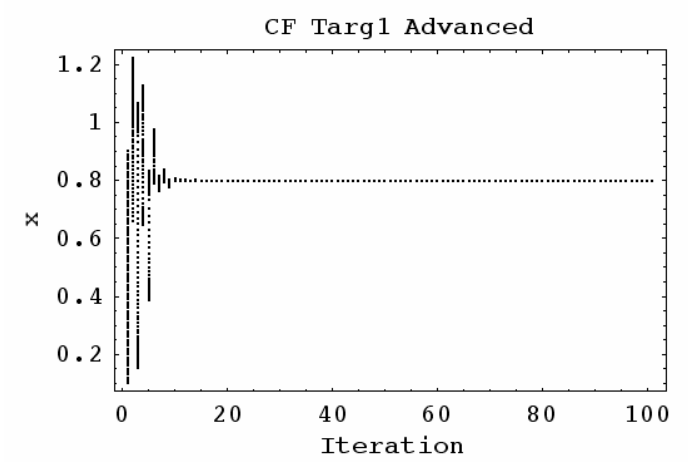

Fig. 4. Best solution: p-1 orbit, $\mathrm{CF}_{\mathrm{T} 1-\mathrm{ADV}}$, SOMA ATAA, complex simulation with $0<x_{\text {initial }}<1,100$ samples

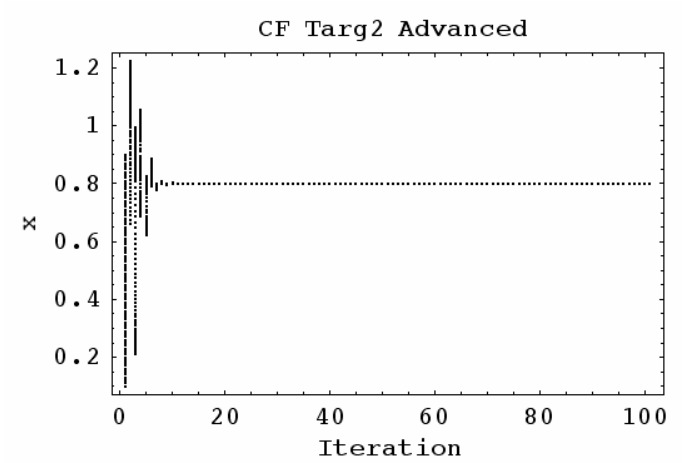

Fig. 5. Best solution: $\mathrm{p}-1$ orbit, $\mathrm{CF}_{\mathrm{T} 2-\mathrm{ADV}}$, SOMA ATAA, complex simulation with $0<x_{\text {initial }}<1,100$ samples

\section{Control of chaos, p-2 orbit}

This case is focused on the stabilization of $\mathrm{p}-2$ orbit. The best results given by $\mathrm{CF}_{\mathrm{T} 1-\mathrm{ADV}}$ (SOMA ATA) and $\mathrm{CF}_{\mathrm{T} 2-\mathrm{ADV}}$ (SOMA ATA) are given in Table 4. The outputs of simulations are depicted in Figures $6-8$.

The results given by $\mathrm{CF}_{\mathrm{T} 1-\mathrm{ADV}}$ show the following attributes: rapid achievement of desired UPO in comparison with $\mathrm{CF}_{\text {Basic }}$ (on average, around 51 iterations are required), together with very poor performance of EA, i.e. the proportion of the solutions with either perfect stabilization or temporary or possibly none at all. Also, relatively considerable period doubling or oscillating in the close neighborhood of desired UPO arose (Fig 7).

In case of $\mathrm{CF}_{\mathrm{T} 2-\mathrm{ADV}}$, the two main above mentioned problems with period doubling (i.e. low-quality 
stabilization) and very poor performance of EAs in finding the stabilizing securing solutions were noticeably suppressed

Table 4: Results for $\mathrm{p}-2$ orbit, $\mathrm{CF}_{\mathrm{T} 1-\mathrm{ADV}}$ and $\mathrm{CF}_{\mathrm{T} 2-\mathrm{ADV}}$

\begin{tabular}{|c|c|c|}
\hline CF Version & $\mathrm{CF}_{\mathrm{TI}-\mathrm{ADV}}$ & $\mathrm{CF}_{\mathrm{T2}-\mathrm{ADV}}$ \\
\hline$K$ & 0.3264 & 0.4208 \\
\hline$F_{\max }$ & 0.1150 & 0.1767 \\
\hline$R$ & 0.1342 & 0.3451 \\
\hline CF Val. & $\mathbf{2 1 6 . 7 9 4 5}$ & $\mathbf{5 . 8 1 \mathbf { 1 0 } ^ { - 9 }}$ \\
\hline Avg. IStab & 51 & 133 \\
\hline
\end{tabular}

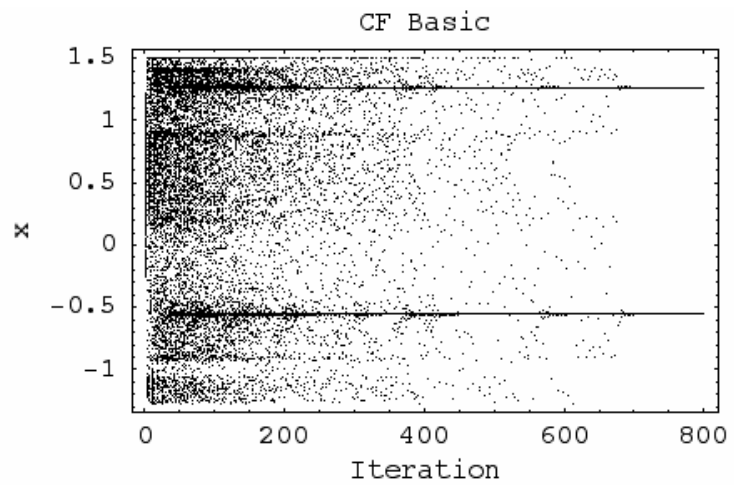

Fig. 6. Best solution: $\mathrm{p}-2$ orbit, $\mathrm{CF}_{\text {Basic }}$, SOMA ATO, complex simulation with $0<x_{\text {initial }}<1,100$ samples

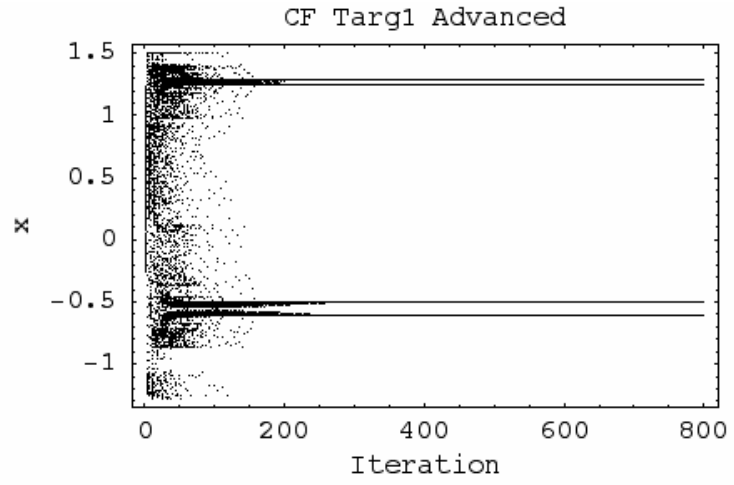

Fig. 7. Best solution: p-2 orbit, $\mathrm{CF}_{\mathrm{T} 1-\mathrm{ADV}}$, SOMA ATA, complex simulation with $0<x_{\text {initial }}<1,100$ samples

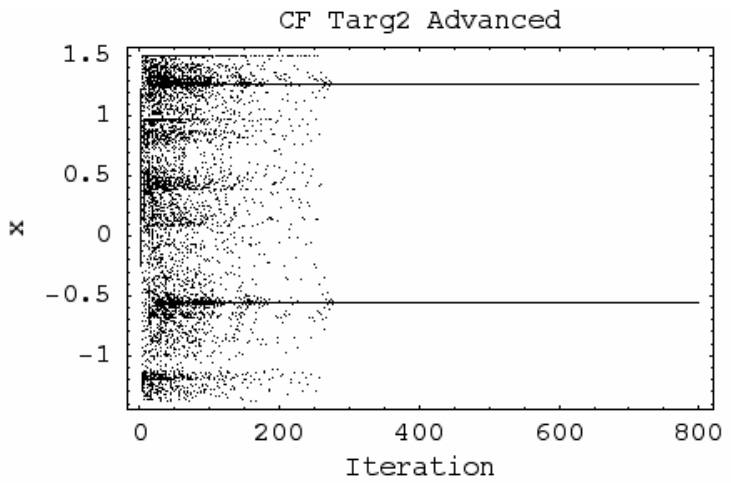

Fig. 8. Best solution: $\mathrm{p}-2$ orbit, $\mathrm{CF}_{\mathrm{T} 2-\mathrm{ADV}}$, SOMA ATA, complex simulation with $0<x_{\text {initial }}<1,100$ samples

\section{Control of chaos, p-4 orbit}

The last case is focused on the stabilization of p-4 orbit. See Table 5 for the results of this optimization. The simulations of the best individual solutions are depicted in Figures 9 - 11.

As a conclusion of this case study, it is possible to say that also in the case of p-4 orbit and optimizations by means of $\mathrm{CF}_{\mathrm{T} 1 \mathrm{ADV}}$ the phenomenon of faster targeting of desired UPO (only 84 iterations) for wide range of initial conditions occurs at the cost of poor performance of EA and period doubling. This is apparent from the notable difference of $\mathrm{CF}$ values given by $\mathrm{CF}_{\mathrm{T1}-\mathrm{ADV}}$ and $\mathrm{CF}_{\mathrm{T} 2 \text {-ADV }}$.

In case of $\mathrm{CF}_{\mathrm{T} 2-\mathrm{ADV}}$, the presented results show positive features as in case of $\mathrm{p}-2$ orbit and from the comparison with $\mathrm{CF}_{\text {Basic }}$ (Fig. 9), it follows that the stabilization was reached very quickly and precisely.

Table 5: Results for $\mathrm{p}-4$ orbit, $\mathrm{CF}_{\mathrm{T} 1-\mathrm{ADV}}$ and $\mathrm{CF}_{\mathrm{T} 2-\mathrm{ADV}}$

\begin{tabular}{|c|c|c|}
\hline CF Version & $\mathrm{CF}_{\mathrm{T} 1-\mathrm{ADV}}$ & $\mathrm{CF}_{\mathrm{T2}-\mathrm{ADV}}$ \\
\hline$K$ & -0.4828 & -0.4154 \\
\hline$F_{\max }$ & 0.1904 & 0.2808 \\
\hline$R$ & 0.4390 & 0.4969 \\
\hline CF Val. & $\mathbf{2 4 1 , 1 8 5 2}$ & $\mathbf{2 . 8 5} \mathbf{1 0}^{-\mathbf{6}}$ \\
\hline Avg. IStab & 84 & 145 \\
\hline
\end{tabular}

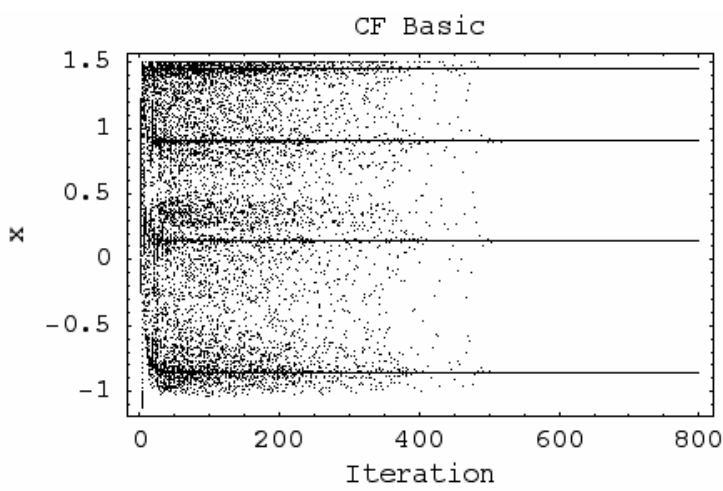

Fig. 9. Best solution: $\mathrm{p}-4$ orbit, $\mathrm{CF}_{\text {Basic }}$, SOMA ATAA, complex simulation with $0<x_{\text {initial }}<1,100$ samples

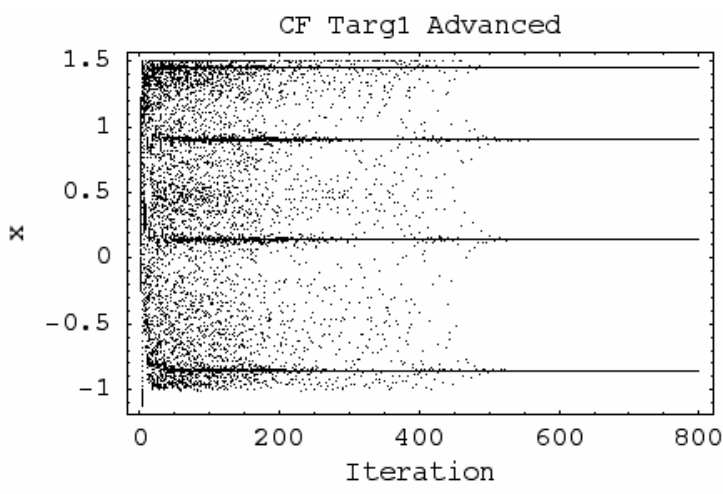

Fig. 10. Best solution: $\mathrm{p}-4$ orbit, $\mathrm{CF}_{\mathrm{T1}-\mathrm{ADV}}$, SOMA ATA, complex simulation with $0<x_{\text {initial }}<1,100$ samples 


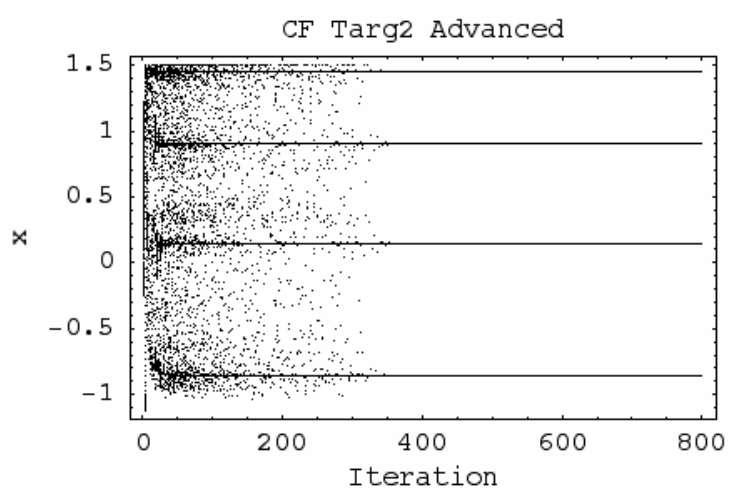

Fig. 11. Best solution: $\mathrm{p}-4$ orbit, $\mathrm{CF}_{\mathrm{T} 2-\mathrm{ADV}}$, SOMA ATO, complex simulation with $0<x_{\text {initial }}<1,100$ samples

\section{Investigation on results of chaos control}

This section presents a accumulation of research (Senkerik et al. 2007) and the results presented here. Please refer to Table 6 for the comparison of average number of iterations required for stabilization, which was elaborated for five CFs and all desired UPOs. Here, a gradual decrease of average IStab value together with development and testing of complex targeting cost functions can be clearly seen.

Table 6: Comparison of results for five CFs

\begin{tabular}{|c|c|c|c|}
\hline UPO & p-1 & p-2 & p-4 \\
\hline CF Basic & 77 & 124 & 122 \\
\hline CF Targ1 & 49 & 113 & 121 \\
\hline CF Targ2 & 39 & 108 & 118 \\
\hline CF Targ1 Adv. & 56 & 51 & 84 \\
\hline CF Targ2 Adv. & 41 & 133 & 143 \\
\hline
\end{tabular}

The first CF Basic gives satisfactory results and can be used wherever the good quality of stabilization is expected and the speed of stabilization and "universality of this solution" for wider range of initial conditions are not decisive.

In case of targeting cost functions, the results for $\mathrm{p}-1$ orbit are significantly better, on the other hand the slightly better results for higher periodic orbits ( $\mathrm{p}-2$ and $\mathrm{p}-4)$ were achieved at the cost of arising of problem with worse performance of EAs and obtaining of solutions with only temporary stabilization or none at all. This negative phenomenon culminates in case of $\mathrm{CF}_{\mathrm{T1}-\mathrm{ADV}}$ where the very low avg. IStab values represents the solutions with rapid and only temporary stabilization.

Finally, $\mathrm{CF}_{\mathrm{T} 2-\mathrm{ADV}}$ design suppress all mentioned problems and gives excellent performance from the point of view of quickness and quality of stabilization for any initial conditions.

\section{CONCLUSION}

The optimization of chaos control described here is relatively simple and easy to implement. Based on obtained results, it may be claimed that all simulations give satisfactory results and thus EAs are capable of solving this class of difficult problems and the quality of results does not depend only on the problem being solved but also on the proper definition of the CF.

From the optimization results it follows, that they are extremely sensitive to the construction of used CF and any small change in the design of $\mathrm{CF}$ can cause radical improvement of the system behavior (as in case of CF Targ2 Advanced), but of course, on the other hand can cause worsening of observed parameters and behavior of chaotic system as well.

All achieved results give the following piece of knowledge.

CF Targ1 advanced is the example of an upgraded CF Targ1 design, which brings the advantage of automatically computed $S C$ value, for the purpose of improving the behavior of controlled chaotic system for wide range of initial conditions. The results were for the first view satisfactory, but two very momentous problems arose - period doubling and very poor performance of EAs. These problems uncovered hidden non-optimal structure of CF Targ1.

In the last proposal of CF Targ2, there were only slight changes in CF design, but from the presented results it can be seen, how such a small change can influence the performance of a controlled system, especially when it is an extremely sensitive chaotic system. CF Targ2 advanced gives excellent results for simulations with wide range of initial conditions and seem to be the choice for the task of finding of "universal and robust solution". The problems with poor EA performance and period doubling were mostly suppressed here. The only disadvantage of this proposal is the relatively big computational-time demands.

Finally, it is hard task to propose a CF, which gives excellent results, especially "universal results" suitable for simulation with wide range of initial conditions. As repeatedly mentioned, the chaotic systems are extremely sensitive to proper settings of control algorithm and of course they are very sensitive to even very tiny change in any parameter. This extreme sensitivity is transferred into complexity of $\mathrm{CF}$ surface thus it is also hard task for EAs to find good solution. It is also difficult to determine the conditions for optimizations and subsequent simulations.

Furthermore parameter settings for EA were based on heuristic approach; therefore there is also possibility for the future research. According to all results showed here it is planned that the main activities will be focused on testing of evolutionary deterministic chaos control in continuous-time and high-order systems and finally testing of evolutionary real-time chaos control. 


\section{ACKNOWLEDGEMENT}

This work was supported by the grant NO. MSM 7088352101 of the Ministry of Education of the Czech republic and by grants of Grant Agency of Czech Republic GACR 102/09/1680.

\section{REFERENCES}

Just W., 1999, "Principles of Time Delayed Feedback Control", In: Schuster H.G., Handbook of Chaos Control, Wiley-Vch, ISBN 3-527-29436-8.

Kwon O.-J., 1999, "Targeting and stabilizing chaotic trajectories in the standard map", Physics Letters A, 258, 229-236.

Ott E., C. Greboki, J.A. Yorke, 1990, "Controlling Chaos", Phys. Rev. Lett. 64, 1196-1199.

Pyragas K., 1992, Continuous control of chaos by selfcontrolling feedback, Physics Letters A, 170, 421-428.

Pyragas K., 1995, "Control of chaos via extended delay feedback", Physics Letters A, 206, 323-330.

Richter H. and K. J. Reinschke, 2000, "Optimization of local control of chaos by an evolutionary algorithm", Physica $D, 144,309-334$.

Richter H., 2002, “An Evolutionary Algorithm for Controlling Chaos: The Use of Multi - Objective Fitness Function", Lecture Notes in Computer Science, vol. 2439, pp. 308320.

Bollt E. M., Kostelich E. J., 1998, „Optimal Targeting of Chaos", Physics Letters A. vol. 245, pp. 399-406.

Kostelich E. J., Ott E., Grebogi C., Yorke J. A., 1993, „Higher-dimensional Targeting“, Physical Review E., vol. 47, No 1, pp. 305-310.

C. M. Bird, P. J. Aston, 1998, "Targeting in the Presence of Noise", Chaos, Solitons, \& Fractals, Volume 9, Issues 12, Pages 251-259.

Ramaswamy R., Sinha S., Gupte N., 1998, „Targeting Chaos Through Adaptive Control“", Physical Review E,. vol. 57, No 3, 1998, pp. 2507-2510.

Starrett J., 2002, „Time-optimal Chaos Control by Center Manifold Targeting“, Physical Review E., vol. 66, No 4, pp. 6206-6211.

S. Iplikci, Y. „Denizhan, 2001, “Control of chaotic systems using targeting by extended control regions method", Physica D: Nonlinear Phenomena, Volume 150, Issues 3-4, 1, Pages 163-176.

S. Iplikci, Y. Denizhan, 2003, „An improved neural network based targeting method for chaotic dynamics", Chaos, Solitons \& Fractals, Volume 17, Issues 2-3, Pages 523529.

Zelinka I., 2004, "SOMA - Self Organizing Migrating Algorithm", In: New Optimization Techniques in Engineering, (B.V. Babu, G. Onwubolu (eds)), chapter 7, 33, Springer-Verlag

Zelinka I., Senkerik R., Navratil E, 2007, "Investigation on Evolutionary Optimitazion of Chaos Control", Chaos, Solitons \& Fractals, doi:10.1016/j.chaos.2007.07.045

Senkerik R., Zelinka I., Navratil E., 2006, "Optimization of feedback control of chaos by evolutionary algorithms", in proc $1^{\text {st }}$ IFAC Conference on analysis and control of chaotic systems, Reims, France, pp 97-102.

Senkerik R., Zelinka I., Navratil E., 2007, "Design of Targeting Cost Function for Evolutionary Optimization of chaos Control", in proc. ECMS 2007, Prague, Czech Republic, pp 345-350.

\section{AUTHORS BIOGRAPHIES}

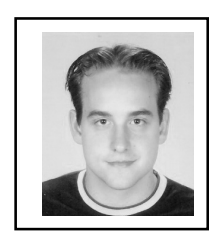

ROMAN SENKERIK was born in the Czech Republic, and went to the Tomas Bata University in Zlin, where he studied Technical Cybernetics and obtained his MSc degree in 2004 and Ph.D. degree in Technical Cybernetics in 2008. He is now a lecturer at the same university (Applied Informatics, Artificial Intelligence, Mathematical Informatics).

Email address: senkerik@fai.utb.cz

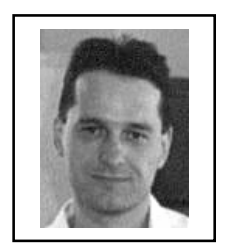

IVAN ZELINKA was born in the Czech Republic, and went to the Technical University of Brno, where he studied Technical Cybernetics and obtained his degree in 1995. He obtained Ph.D. degree in Technical Cybernetics in 2001 at Tomas Bata University in Zlin. Now he is an associated professor (Artificial Intelligence, Theory of Information) and head of department.

Email address: zelinka@fai.utb.cz .

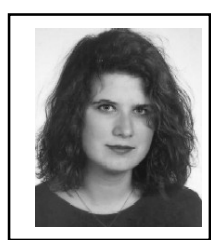

ZUZANA OPLATKOVA was born in Czech Republic, and went to the Tomas Bata University in Zlin, where she studied technical cybernetics and obtained her MSc. degree in 2003 and Ph.D. degree in 2008. She is a lecturer (artificial intelligence) at the same university. Her email address is: oplatkova@fai.utb.cz 